\title{
Modelagem simplificada para estimativa do potencial de penetração de partículas em substratos porosos
}

\author{
A simplified model to estimate the penetration potential \\ of particles in porous substrates
}

Eliane Betânia Carvalho Costa Departamento de Engenharia de Construção Civil, Escola Politécnica Universidade de São Paulo Avenida Prof. Almeida Prado, trav. 2, n.83, Edif. de Eng. Civil, Laboratório de Microestrutura e Ecoeficiência, Sala S41, Cidade Universitária São Paulo - SP - Brasil CEP 05508-900 Tel.: (11)30915166 E-mail: eliane.costa@lme.pcc.usp.br

Fernando Akira Kurokawa Departamento de Engenharia de Construção Civil, Escola Politécnica Universidade de São Paulo Tel.: (11) 3091-5388 E-mail:

fernando.kurokawa@poli.usp.br

Vanderley Moacyr John Departamento de Engenharia de Construção Civil, Escola Politécnica Universidade de São Paulo Tel.: (11) 3091-5794 E-mail: vmjohn@lme.pcc.usp.br

Recebido em 22/04/10/12 Aceito em 10/03/13

\section{Eliane Betânia Carvalho Costa \\ Fernando Akira Kurokawa Vanderley Moacyr John}

\section{Resumo}

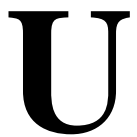

m modelo simplificado foi desenvolvido para estimar o potencial de penetração de partículas típicas de matrizes de argamassas e concretos nos poros de um substrato. $\mathrm{O}$ modelo considera que, após serem lançadas sobre a superfície, as partículas com área de projeção no plano menor ou igual à área do poro podem penetrá-la. Foram estudadas cinco matrizes distintas, constituídas por partículas de cimento, cal, filer calcário, microssílica e argamassa aplicadas em três substratos com distribuição do tamanho de poros similares aos encontrados no mercado nacional. A distribuição granulométrica das partículas foi obtida por ensaios de granulometria a laser e a distribuição do tamanho dos poros e a porosidade dos substratos por porosimetria de intrusão de mercúrio. Os resultados obtidos mostraram que o potencial penetração de partículas nos poros não atinge $1 \%$ do volume total de partículas presentes na matriz, mesmo para a matriz com granulometria mais fina (microssílica) em substrato com faixa de poros mais ampla (substrato cimentício) numa relação área do poro/área da partícula igual a 1. Conforme modelo proposto, a contribuição da parcela mecânica pela penetração de partículas nos poros para aderência é insignificante.

Palavras-chave: Aderência mecânica. Partículas. Pporos. Filtração. Modelagem.

\section{Abstract}

A simplified model was developed to estimate the penetration potential of particles typical of mortars and concrete into the pores of different substrates. This model considers that particles with projected area less than or equal to the pore area can penetrate the pore. Five suspensions made with particles of cement, hydrated lime, ground limestone, microsilica, and a typical cement mortar were applied in three different substrates with different pore size distributions, similar to substrates found in the market. The particle size distribution was obtained by laser diffraction granulometry, while the pore size distribution and the porosity of substrates were measured by mercury intrusion porosimetry. The results showed that the potential penetration of particles in the pores is less than $1 \%$ of the total volume of particles, even for the suspensions with finer particle size (microsilica) on a substrate with larger pores (cementitious substrate), considering a pore area/particle area ratio of 1 . The model showed that the mechanical contribution to the penetration of the particles into the pores is negligible.

Keywords: Mechanical adhesion. Particles. Pores. Filtration. Modeling. 


\section{Introdução}

Quando uma suspensão entra em contato com uma superfície porosa, parte da fase líquida, que inclui eventuais íons dissolvidos das partículas, pode penetrar no interior dos poros da base se as forças capilares forem positivas. No caso de suspensões cimentícias, existem evidências qualitativas da presença de compostos hidratados e elementos químicos típicos do cimento nas superfícies dos poros (DÉTRICHÉ et al., 1984; DUPIN; DETRICHE; MASO, 1988; CARASEK, 1996; CARVALHO JÚNIOR, 2005) - essas espécies químicas podem ser resultado da precipitação dos íons dissolvidos na água do poro ou até mesmo da penetração de partículas. Tais evidências têm sido a principal constatação de que a precipitação de hidratos dos ligantes dentro dos poros da base constitui-se no mecanismo fundamental da aderência pasta-base.

No entanto, dada a dificuldade experimental, até o momento, não existe uma quantificação do material que penetra nos poros, tampouco uma comprovação (ou negação) direta que esses produtos hidratados os colmatem. Além disso, para que haja contribuição para aderência, o material nos poros deve apresentar propriedades mecânicas adequadas.

Sendo assim, a contribuição dos produtos depositados nos poros para a aderência, medida por tração direta, depende da quantidade desses produtos (fração de área e/ou volume que eles cobrem) e de sua resistência mecânica, que nos ligantes é controlada principalmente pela porosidade, sendo essa uma função da relação água-ligante que governa o excesso de água.

A precipitação de compostos hidratados depende da concentração de íons na solução. Se não houver a penetração de partículas nos poros do substrato, a matriz será filtrada. Desse modo, a concentração de íons da água absorvida pelos poros seria inferior à da matriz aplicada (a/c variando de 1,14 a 9,26, dependendo do tipo de cimento) (COSTA; JOHN, 2011). A baixa relação $\mathrm{a} / \mathrm{c}$ levaria à formação de produtos hidratados com elevada porosidade e, consequentemente, com baixa resistência. Além disso, a porosidade típica de materiais cerâmicos está na faixa de 20-30\%, e de concretos, de 10 $15 \%$. Mesmo considerando que todos os poros sejam totalmente colmatados, a contribuição para a resistência certamente seria pequena.

Assim, a única hipótese que permitiria um ganho de resistência significativo seria a penetração de partículas de ligante dentro dos poros, aumentando a área de contato substrato-matriz cimentícia. A penetração pode ocorrer se o diâmetro da partícula (ou aglomerados de partículas) for menor que o do poro (restrições geométricas) e se existirem forças capazes de mover a partícula na direção do mesmo. Entre essas forças destacamos a energia de aplicação da suspensão; a força da gravidade; o arraste hidrodinâmico (movimentação da água); e atração (ou repulsão) eletrostática das partículas pela superfície do meio poroso. Não se trata de algo homogêneo. O efeito da força da gravidade depende da posição relativa entre a base e a suspensão (ou os poros e as partículas). As forças capilares dependem da geometria dos poros e da molhabilidade, sendo essa característica dependente do ângulo de contato, que pode ser uma função do tempo.

A restrição geométrica tem sido amplamente abordada pela área de processos e mecanismos de filtração. Alguns modelos matemáticos foram formulados para estimar a quantidade de partículas que pode penetrar nos orifícios e, com isso, prever a eficiência do processo de filtração (REGE; FOGLER, 1987; SHARMA; YORTSOS, 1987; IMDAKM; SAHIMI, 1987; KAISER, 1997; BORISOVA; ADLER, 2005; KAMPEL, 2007; ROUSSEL; NGUYEN; COUSSOT, 2007; CHEVOIR; GAULARD; ROUSSEL, 2007; MAYS, 2010). O modelo de restrição geométrica também tem outra aplicação prática, pois permite entender o efeito da rugosidade superficial nos processos de aderência e formação da zona de transição. Adicionalmente, o modelo pode fornecer diretrizes para otimizar a porosidade do substrato, de forma a propiciar ganho de aderência.

Roussel e outros mostraram experimentalmente que o entupimento dos orifícios em peneiras é uma questão de probabilidade e está relacionado ao tamanho da partícula, à concentração de sólidos no sistema (que controla a probabilidade de que mais de uma partícula possa entrar simultaneamente em um orifício) e à distribuição do tamanho dos orifícios. Os autores verificaram que, à medida que se aumenta a relação entre o diâmetro do furo e o da partícula, maior é a quantidade de partículas retidas na peneira; isso ocorre mesmo em sistemas com concentrações muito baixas (10\%). Além disso, observaram que mesmo partículas dispersas muito menores que os orifícios podem ser retidas, quando várias partículas chegam ao mesmo tempo em um mesmo orifício (ROUSSEL; NGUYEN; COUSSOT, 2007). No entanto, ainda não existe aplicação desses conceitos na área de contato de suspensões cimentícias com bases porosas.

O presente trabalho tem como objetivo estimar o potencial de penetração das partículas presentes numa matriz de ligantes típicos de argamassas e de 
concretos aplicados em substratos com diferentes distribuições de poros comuns ao mercado nacional. Desse modo, será possível verificar se a quantidade de material que penetra nos poros constitui-se no principal mecanismo de aderência matriz-substrato.

\section{Formulação do modelo simplificado}

O modelo elaborado para estimar o potencial de penetração das partículas presentes em uma matriz nos poros do substrato considera que a(s) partícula(s) que tenha(m) área de projeção no plano menor ou igual à área de projeção do poro poderá(ão) penetrá-lo.

Em um mesmo poro podem penetrar partículas de um único tamanho (Figuras 1a e 1b) ou de tamanhos diferentes (Figura 1c), e desprezam-se o efeito da forma e a profundidade do poro.

Quanto maior a concentração de partículas, menor é a distância entre as partículas, o que tende a intensificar a aglomeração delas, tornando a distribuição granulométrica efetiva de partículas mais grosseira (RIPPERGER, 2002; ROUSSEL; NGUYEN; COUSSOT, 2007). No caso do cimento, além de esse efeito ser intensificado, com o avanço da hidratação as partículas alteram sua forma e dimensão devido aos mecanismos de dissolução-precipitação.

Estudos em soluções altamente diluídas mostram que, após 30 min do contato com água ou água saturada em íons cálcio, as partículas de cimento aumentam de diâmetro (SILVA; MONTEIRO, 2005; CARDOSO, 2010; JUILLAND et al., 2010).

Juilland et al. (2010) verificaram, por análise de imagens (MEV), que não houve a formação de hidratos na superfície da alita após 2 min de contato com água e observaram pequenos pontos de lixiviação (pites). Esse efeito foi mais intenso após 30 min de hidratação, com maior proporção e maior dimensão dos pontos de lixiviação. Os autores observaram a formação de hidratos na superfície da alita somente após o contato desta com água saturada em cal.

Nesse modelo, esses fatores foram desprezados, ou seja, o modelo não considera os efeitos de aglomeração; todas as partículas estão perfeitamente dispersas e não há alteração de volume das partículas em função do tempo de contato com a água.

Em outras palavras, o modelo proposto apresenta o potencial máximo de penetração e, na prática, superestima a capacidade real de penetração. Adicionalmente, para a elaboração do modelo foram feitas as seguintes considerações:

(a) a velocidade das partículas no fluido foi considerada constante e independente do tamanho da partícula, de tal modo que todas as partículas tinham a mesma chance de chegar ao poro;

(b) embora existam forças capazes de mover as partículas na direção dos poros (ex.: fluxos de água causados por absorção), forças hidrodinâmicas e eletrostáticas e os efeitos inerciais foram desprezados. O modelo não considera qualquer tipo de força em particular: assume-se que todas as partículas se movem para os poros, de modo a garantir o máximo de penetração (alocação ótima). Essa hipótese é possível, embora improvável;

(c) a energia de superfície e a rugosidade do substrato foram negligenciadas;

(d) a profundidade do poro e a conexão entre eles não foram consideradas; e

(e) os poros são cilíndricos, e as partículas são esféricas.

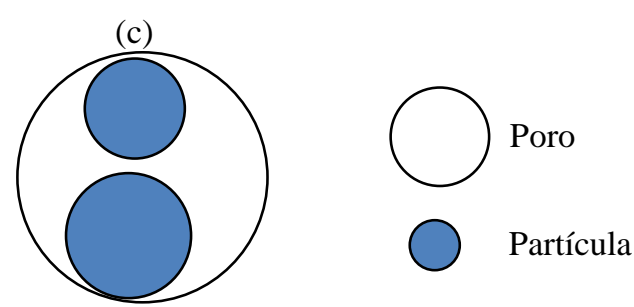

Figura 1 - Partículas com área de projeção igual ou inferior à área de projeção do poro podem penetrálo

Nota: Assim, num mesmo poro pode haver penetração de uma (Figura 1a) e/ou mais partículas (Figuras 1b e 1c), podendo estas ser de mesmos tamanhos (Figura 1b) ou de tamanhos diferentes (Figura 1c). 


\section{Estimativa do potencial de penetração de partículas em substratos porosos}

O modelo proposto considera as condições a seguir.

Para que haja penetração de partículas nos poros (Equação 1):

$\mathrm{A}_{\text {po }} \geq n \mathrm{~A}_{\text {pa }}$

Eq. 1

Em que:

$\mathrm{A}_{\mathrm{po}}$ é a área do poro;

$\mathrm{A}_{\mathrm{pa}}$ é a área da partícula; e

$n$ é a relação entre o diâmetro do poro e o diâmetro da partícula.

Se $n=1$, penetram no poro todas partículas cuja área de projeção no plano seja menor ou igual à área de projeção do poro. Mc-Dowell-Boyer, Hunt e Sitar (1986), após uma ampla revisão bibliográfica, verificaram que $n$ varia de 4 a 20 , hipótese que será investigada posteriormente (Figuras 4 e 5).

Se $\mathrm{A}_{\mathrm{po}}<n \mathrm{~A}_{\mathrm{pa}}$, não ocorre penetração de partículas nos poros do substrato.

Assim, se $\mathrm{A}_{\mathrm{po}} \geq n \mathrm{~A}_{\mathrm{pa}}$ a probabilidade de uma partícula encontrar um poro no momento em que se aproxima da superfície $\left(\mathrm{P}^{*}\right)$ é calculada em função da fração de partículas (fai), da fração de poros (foi) de um dado diâmetro e da porosidade do substrato (p), como mostra a Equação 2:

$\mathrm{P}^{*}=\sum_{\mathrm{i}}\left(\mathrm{fa}_{\mathrm{i}} \times \mathrm{fo}_{\mathrm{i}} \times \mathrm{p}\right)$

Eq. 2

Em que:

$\mathrm{fa}_{\mathrm{i}}$ é obtida da seguinte forma (Equação 3):

$\mathrm{fa}_{\mathrm{i}}=\frac{\mathrm{Na}_{\mathrm{i}}}{\mathrm{N}_{\mathrm{t}}}$

Eq. 3

Na qual:

$\mathrm{Na}_{\mathrm{i}}$ é o número de partículas de dado diâmetro; e

$\mathrm{N}_{\mathrm{t}}$ é o número total de partículas necessário para recobrir a superfície do substrato.

Neste trabalho, a fração de poros em cada diâmetro $\left(\mathrm{fo}_{\mathrm{i}}\right)$ e a porosidade total do substrato $(\mathrm{p})$ foram obtidas pelo ensaio de porosimetria por intrusão de mercúrio.

O número total de partículas é calculado com a seguinte expressão (Equação 4):

$\mathrm{N}_{\mathrm{t}}=\sum_{\mathrm{i}} \frac{6 \mathrm{~A}_{\mathrm{s}} \alpha \mathrm{e}}{\pi \mathrm{da}_{\mathrm{i}}^{3}}$

Eq. 4

Em que:

$\mathrm{A}_{\mathrm{s}}$ é a área do substrato a ser revestida;

$\alpha$ é a concentração de partículas da matriz; e é a espessura da camada que poderá contribuir com partículas; e

$\mathrm{da}_{\mathrm{i}}$ é o diâmetro de cada partícula.

$\mathrm{O}$ número de partículas de dado diâmetro $\left(\mathrm{Na}_{\mathrm{i}}\right)$ é calculado multiplicando-se o número total de partículas $\left(\mathrm{N}_{\mathrm{t}}\right)$ pela frequência de partículas daquele diâmetro $\left(\mathrm{fa}_{\mathrm{i}}\right)$, conforme a Equação 5:

$\mathrm{Na}_{\mathrm{i}}=\mathrm{N}_{\mathrm{t}} \times \mathrm{fa}_{\mathrm{i}}$.

Eq. 5

A distribuição de frequência das partículas em cada diâmetro pode ser obtida, entre outros métodos, por granulometria a laser.

Tem-se, ainda, que o volume de partículas que pode penetrar nos poros depende do volume de poros disponíveis e do volume de partículas com área de projeção menor ou igual à área de cada poro. Por exemplo, se o volume de partículas for superior ao volume de poros, o volume de partículas que poderá penetrá-lo será no máximo igual ao volume dos poros. Desse modo, o volume de poros $\left(\mathrm{Vo}_{\mathrm{i}}\right)$ e o volume das partículas que pode penetrar no poro $\left(\mathrm{V}_{i}^{*}\right)$ são calculados pelas Equações 6 e 7 respectivamente:

$\mathrm{Vo}_{\mathrm{i}}=\mathrm{A}_{\mathrm{s}} \times \mathrm{e}_{\mathrm{s}} \times \mathrm{fo}_{\mathrm{i}}$

Eq. 6

$\mathrm{V}_{\mathrm{i}}^{*}=\mathrm{Na}_{\mathrm{i}} \times \mathrm{Va}_{\mathrm{i}} \times \mathrm{P}^{*}$

Eq. 7

Em que:

$\mathrm{e}_{\mathrm{s}}$ é a espessura do substrato; e

$\mathrm{Va}_{\mathrm{i}}$ é o volume de cada partícula.

Se as partículas da matriz foram tomadas como esferas perfeitas não reativas, o volume de dada partícula $\left(\mathrm{Va}_{\mathrm{i}}\right)$ é dado por (Equação 8):

$\mathrm{Va}_{\mathrm{i}}=\frac{\pi \mathrm{da}_{\mathrm{i}}^{3}}{6}$

Eq. 8

Com base nas Equações 6 e 7, observa-se que se:

$\mathrm{Vo}_{\mathrm{i}}<\mathrm{V}_{\mathrm{i}}^{*}$, o volume máximo de partículas que penetra nos poros $\left(\overline{\mathrm{V}}_{\mathrm{i}}\right)$ é igual à $\mathrm{Vo}_{\mathrm{i}} ; \mathrm{e}$

$\mathrm{Vo}_{\mathrm{i}} \geq \mathrm{V}_{\mathrm{i}}^{*}$, o volume de partículas que poderá penetrar nos poros $\left(\overline{\mathrm{V}}_{\mathrm{i}}\right)$ será igual a $\mathrm{V}_{\mathrm{i}}^{*}$.

Portanto, o potencial máximo de penetração de partículas em substratos porosos $\left(\mathrm{P}_{\max }\right)$ é o somatório dos volumes que penetram em cada diâmetro $\left(\bar{V}_{i}\right)$ dividido pelo volume total de partículas $\left(\mathrm{V}_{\mathrm{t}}\right)$ necessário para recobrir o substrato, como mostra a Equação 9:

$\mathrm{P}_{\text {máx }}=\frac{\sum_{\mathrm{i}} \overline{\mathrm{V}}_{\mathrm{i}}}{\mathrm{V}_{\mathrm{t}}} \times 100$

Eq. 9

A porcentagem de poros preenchidos pelas partículas $(\overline{\mathrm{P}})$ é calculada dividindo-se o volume de partículas $\left(\overline{\mathrm{V}}_{\mathrm{i}}\right)$ que penetra pelo volume total de poros do substrato $\left(\mathrm{Vo}_{\mathrm{t}}\right)$, conforme a Equação 10: 
$\overline{\mathrm{P}}=\frac{\sum_{\mathrm{i}} \overline{\mathrm{V}}_{\mathrm{i}}}{\mathrm{Vo}_{\mathrm{t}}} \times 100$

Eq. 10

\section{Aplicação do modelo}

O potencial de penetração de partículas em meios porosos foi avaliado para partículas típicas de argamassas e concretos em substratos com diferentes distribuições de poros, todos disponíveis no mercado nacional. Foram feitas as análises da influência da distribuição granulométrica das partículas, da distribuição do tamanho de poros e da relação entre o diâmetro do poro e o da partícula.

Para tanto, no estudo de caso, consideraram-se distribuições de tamanho de partículas de ligantes (cimento e cal), de adições minerais e pozolânicas (filer calcário e microssílica), e de uma argamassa (constituída por cimento, cal e areia) e de três substratos (superfície de argamassa - SCIM-A - e dois cerâmicos - SCER-B e S-CERC) com distribuição de tamanhos de poros distintos.

A distribuição granulométrica das partículas foi obtida por granulometria a laser (equipamento Malvern Mss Mastersizer) utilizando-se água deionizada como meio dispersante. Utilizou-se água deionizada porque o tempo de realização do ensaio é inferior a $1 \mathrm{~min}$. Assumiu-se, com base nos resultados de Juilland et al. (2010), que esse período não altera significativamente a dimensão das partículas de cimento. A distribuição granulométrica da argamassa foi realizada em duas etapas: peneiramento mecânico para a obtenção das frações compreendidas entre 4,0 mm e $90 \mu \mathrm{m}$; e granulometria a laser, para aquisição da fração fina (partículas inferiores a $90 \mu \mathrm{m}$ ).

A distribuição do tamanho de poros dos substratos foi medida por porosimetria de intrusão de mercúrio (porosímetro Micromeritics - Autopore III 9420), assumindo-se como condições de ensaio:

(a) tensão superficial do mercúrio igual a 0,485 $\mathrm{N} / \mathrm{m}$; densidade do mercúrio de $13,53 \mathrm{~g} / \mathrm{cm}^{3}$; e

(b) ângulo de contato mercúrio/sólido de $130^{\circ}$.

Ambos os resultados estão apresentados, respectivamente, nas Figuras 2 e 3.

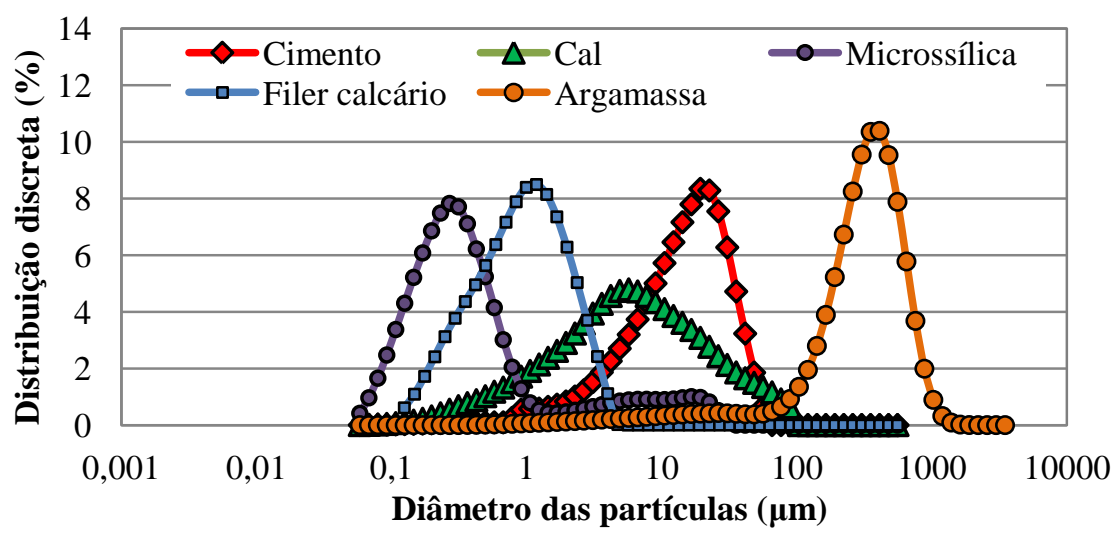

Figura 2 - Distribuição discreta das partículas no primeiro minuto de contato com a água

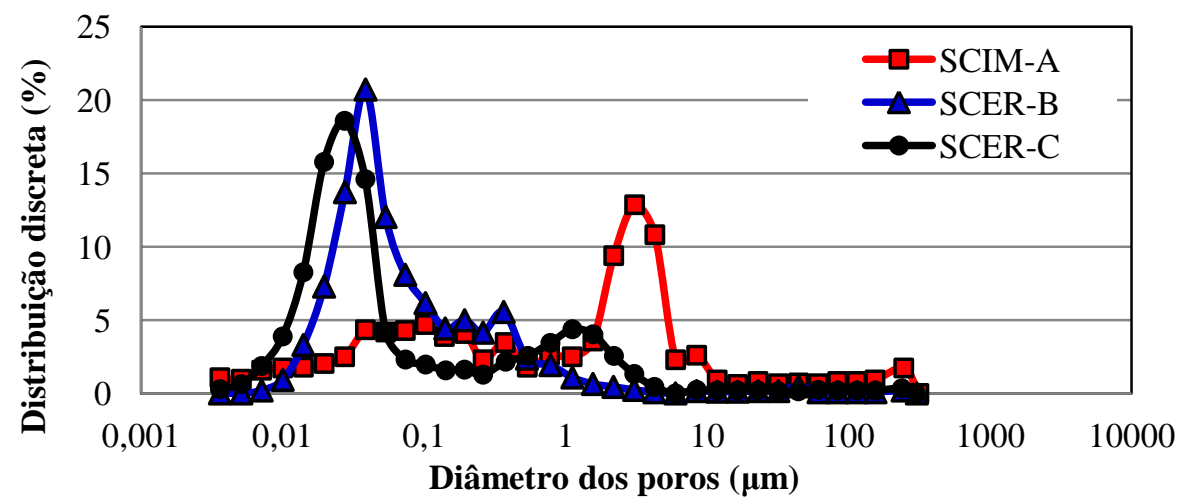

Figura 3 - Distribuição discreta de poros dos substratos analisados 
Sabe-se que as partículas de cimento ampliam sua forma e dimensão com o avanço da hidratação. No entanto, admitiu-se que essas eram esferas perfeitas e sem alteração de volume no decorrer do tempo. As análises foram realizadas com distribuição granulométrica das partículas no primeiro minuto de contato da água.

A porosidade dos substratos analisados também foi obtida pelo ensaio de porosimetria de intrusão de mercúrio, sendo igual a $34,4 \%, 41,6$ e $36,1 \%$ para os substratos SCIM-A, SCER-B e SCER-C respectivamente.

O número de partículas foi calculado considerando-se a área do substrato a ser revestida igual a $10.000 \mu \mathrm{m}^{2}(1=100 \mu \mathrm{m}$, valor arbitrário $)$; espessura de $50 \mu \mathrm{m}$ (segundo alguns autores ${ }^{1}$, essa é a espessura máxima da zona de transição em concretos), e a concentração de partículas de 57\%, conforme resultados obtidos por Cardoso em estudo abrangente de formulações de argamassas (CARDOSO, 2009).

O modelo foi implementado em planilha Excel.

\section{Resultados e discussão}

A Tabela 1 apresenta o potencial máximo de penetração de partículas com diferentes distribuições granulométricas presentes em dada matriz em substratos com porosidade $\mathrm{e}$ distribuição de poros distintos.

Observa-se na Tabela 1 que, entre os substratos analisados, o maior potencial de penetração ocorre em substratos cimentícios, pois estes apresentam uma faixa de distribuição de poros com diâmetro entre $1 \mu \mathrm{m}$ e $10 \mu \mathrm{m}$ superior aos cerâmicos. As pastas de microssílica e filer calcário apresentam maior potencial de penetração em relação às outras partículas analisadas, pois possuem uma distribuição granulométrica mais fina.

O potencial de penetração das matrizes de argamassa e cimento é equivalente, isso porque a fração fina da argamassa é constituída essencialmente por cimento. Nota-se que não há penetração dessas partículas nos poros do substrato cerâmico, ou seja, as partículas presentes apresentam diâmetro superior ao dos poros do substrato.

Matrizes de cal altamente diluídas têm sido utilizadas na restauração de patrimônios históricos com o objetivo de reduzir a porosidade das argamassas pela penetração das partículas nos poros e, posteriormente, a precipitação de carbonato de cálcio. Os resultados do modelo

${ }^{1}$ Scrivener, Crumbie e Laugesen, 2004 e Stroeven e Hu, 2009. mostram pequena capacidade de penetração da cal nos poros do substrato, tal como sugerem alguns pesquisadores (BAGLIONI; GIORGI, 2006; DANIELE; TAGLIERI; QUARESIMA, 2008), que relatam que a principal dificuldade na consolidação tem sido a baixa capacidade de penetração da cal (cerca de $1 \mathrm{~mm}$ ). Além disso, a baixa concentração de cal nessas suspensões também dificulta a consolidação, ou seja, o aumento da coesão da matriz pela recuperação do ligante perdido ou degradado (TAVARES, VEIGA, 2011).

Segundo a teoria de filtração, o fator que determina a penetração de partículas em meios porosos é a relação entre o diâmetro do orifício $\left(d_{o}\right)$ e o das partículas $\left(d_{a}\right)$; essa relação é denominada $n$. Tal como mencionado anteriormente, a migração de partículas em meios porosos só irá ocorrer se o seu for inferior ao diâmetro do orifício, ou seja, $n \geq 1$. Se $n<1$, haverá a obstrução do orifício e o acúmulo de partículas na superfície. Sakthivadiveld $\left(1967^{2}\right.$ apud McDOWELL-BOWYER; HUNT; SITAR, 1986) verificou experimentalmente que não ocorre a penetração de partículas plásticas imersas em óleo mineral em orifícios:

(a) mesmo que estas possuam uma relação $n<$ 10, a matriz é filtrada;

(b) para $10<n<20$, ocorre a deposição das partículas na superfície e uma redução da permeabilidade de filtração; e

(c) para $n>20$, somente $2-5 \%$ dos orifícios são preenchidos pelas partículas.

O potencial de penetração das partículas presentes nas matrizes estudadas foi calculado para o substrato cimentício considerando-se os seguintes valores de $n: 1,4,10,20,30,40,50$ e 100. Os resultados são mostrados na Figura 4.

O potencial de penetração das partículas reduz-se com o aumento de $n$ numa correlação logarítmica entre os dois parâmetros. Para $n=10$, o potencial de penetração das matrizes de argamassa, de cimento e de cal é reduzido em 50\% (Figura 4a); e reduz-se em $40 \%$ e $30 \%$ para as matrizes de filer calcário e microssílica respectivamente (Figura 4b).

Na Figura 5 é apresentada a distribuição de partículas de microssílica que penetram nos poros dos substratos considerando a relação entre os diâmetros poros/partícula: $n=1$ e $n=10$.

\footnotetext{
${ }^{2}$ Sakhthivadivel, R. Theory and Mechanism of Filtration of Non-Colloidal Fines Through a Porous Medium. Berkeley: University of California, 1967. 220 p.
} 
Tabela 1 - Potencial máximo de penetração de partículas presentes numa matriz $\left(\mathbf{P}_{\text {máx }}\right)$ e a porcentagem de poros preenchidos por partículas nos poros $(\overline{\mathbf{P}})$ de diferentes substratos

\begin{tabular}{|c|c|c|c|c|c|c|c|c|}
\hline Substrato & Matriz & $\begin{array}{c}V_{t}^{(1)} \\
\left(\mathbf{c m}^{3}\right)\end{array}$ & $\begin{array}{l}\mathrm{Vo}_{\mathrm{t}}{ }^{(1)} \\
\left(\mathrm{cm}^{3}\right)\end{array}$ & $\begin{array}{l}\overline{\mathbf{V}}_{\mathbf{i}}^{(1)} \\
\left(\mathbf{c m}^{3}\right)\end{array}$ & $\begin{array}{l}P_{\text {máx }} \\
(\%)\end{array}$ & $\begin{array}{c}\overline{\mathbf{P}} \\
(\%)\end{array}$ & $\begin{array}{c}\mathbf{M}_{\mathbf{t}}^{(2)} \\
(\mathbf{g})\end{array}$ & $\begin{array}{l}A_{c}^{(1)} \\
(\%)\end{array}$ \\
\hline \multirow{5}{*}{$\begin{array}{c}\text { SCIM-A } \\
\mathbf{p}=\mathbf{3 4 , 4 \%}\end{array}$} & Argamassa & $1,86 \times 10^{-3}$ & $3,44 \times 10^{-5}$ & $1,84 \times 10^{-7}$ & 0,01 & 0,54 & $5,21 \times 10^{-7}$ & 0,19 \\
\hline & Cimento & $2,85 \times 10^{-3}$ & $3,44 \times 10^{-5}$ & $2,47 \times 10^{-7}$ & 0,01 & 0,72 & $7,13 \times 10^{-7}$ & 0,25 \\
\hline & Cal & $2,85 \times 10^{-3}$ & $3,44 \times 10^{-5}$ & $1,69 \times 10^{-6}$ & 0,06 & 4,90 & $3,81 \times 10^{-6}$ & 1,69 \\
\hline & Fíler calcário & $2,85 \times 10^{-3}$ & $3,44 \times 10^{-5}$ & $1,46 \times 10^{-5}$ & 0,51 & 42,52 & $3,83 \times 10^{-5}$ & 14,63 \\
\hline & Microssílica & $2,85 \times 10^{-3}$ & $3,44 \times 10^{-5}$ & $2,15 \times 10^{-5}$ & 0,75 & 62,44 & $4,72 \times 10^{-5}$ & 21,48 \\
\hline \multirow{5}{*}{$\begin{array}{c}\text { SCER-B } \\
\mathbf{p}=\mathbf{4 1 , 6 \%}\end{array}$} & Argamassa & $1,86 \times 10^{-3}$ & $4,16 \times 10^{-5}$ & $2,05 \times 10^{-8}$ & 0,00 & 0,05 & $5,21 \times 10^{-8}$ & 0,02 \\
\hline & Cimento & $2,85 \times 10^{-3}$ & $4,16 \times 10^{-5}$ & $8,76 \times 10^{-8}$ & 0,00 & 0,21 & $2,53 \times 10^{-7}$ & 0,09 \\
\hline & Cal & $2,85 \times 10^{-3}$ & $4,16 \times 10^{-5}$ & $4,88 \times 10^{-7}$ & 0,02 & 1,17 & $1,10 \times 10^{-6}$ & 0,49 \\
\hline & Fíler calcário & $2,85 \times 10^{-3}$ & $4,16 \times 10^{-5}$ & $4,51 \times 10^{-6}$ & 0,16 & 10,86 & $1,18 \times 10^{-5}$ & 4,52 \\
\hline & Microssílica & $2,85 \times 10^{-3}$ & $4,16 \times 10^{-5}$ & $9,66 \times 10^{-6}$ & 0,34 & 23,23 & $2,12 \times 10^{-5}$ & 9,66 \\
\hline \multirow{5}{*}{$\begin{array}{c}\text { SCER-C } \\
\mathbf{p}=\mathbf{3 6 , 1 \%}\end{array}$} & Argamassa & $1,86 \times 10^{-3}$ & $3,61 \times 10^{-5}$ & $5,18 \times 10^{-8}$ & 0,00 & 0,14 & $1,47 \times 10^{-7}$ & 0,05 \\
\hline & Cimento & $2,85 \times 10^{-3}$ & $3,61 \times 10^{-5}$ & $9,27 \times 10^{-8}$ & 0,00 & 0,26 & $2,68 \times 10^{-7}$ & 0,09 \\
\hline & $\mathrm{Cal}$ & $2,85 \times 10^{-3}$ & $3,61 \times 10^{-5}$ & $6,44 \times 10^{-7}$ & 0,02 & 1,79 & $1,46 \times 10^{-6}$ & 0,65 \\
\hline & Fíler calcário & $2,85 \times 10^{-3}$ & $3,61 \times 10^{-5}$ & $5,76 \times 10^{-6}$ & 0,20 & 15,97 & $1,51 \times 10^{-5}$ & 5,77 \\
\hline & Microssílica & $2,85 \times 10^{-3}$ & $3,61 \times 10^{-5}$ & $8,79 \times 10^{-6}$ & 0,31 & 24,37 & $1,93 \times 10^{-5}$ & 8,80 \\
\hline
\end{tabular}

Nota: adotando os seguintes parâmetros: $\alpha=57 \% ; e=50 \mu \mathrm{m} ; \mathrm{A}_{\mathrm{s}}=1.000 .000 \mu \mathrm{m}^{2} ; \mathrm{e}_{\mathrm{s}}=100 \mu \mathrm{m} ; \mathrm{e}, n=1$.

${ }^{(1)} V_{t}$ - Volume total de partículas da matriz; $V_{t}$ - Volume total de poros do substrato; $\bar{V}_{i}$ - Volume de partículas que penetra nos poros; e $\mathrm{A}_{c}$ - Acréscimo da área de contato; $\mathrm{e}$

${ }^{(2)}$ Massa das partículas nos poros $\left(\mathrm{M}_{\mathrm{t}}\right)$ calculada multiplicando-se a densidade das partículas $(\rho)$ pelo volume destas nos poros $\left(\overline{\mathrm{V}}_{\mathrm{i}}\right)$.

A densidade das partículas foi obtida por picnometria a gás hélio, sendo pargamassa $=2,83 \mathrm{~g} / \mathrm{cm}^{3} ; \rho$ cimento $=2,89 \mathrm{~g} /$ $\mathrm{cm}^{3} ; \rho \mathrm{cal}=2,26 \mathrm{~g} / \mathrm{cm}^{3} ;$ filer calcário $=2,62 \mathrm{~g} / \mathrm{cm}^{3} ;$ e pmicrossilia $=2,20 \mathrm{~g} / \mathrm{cm}^{3}$.

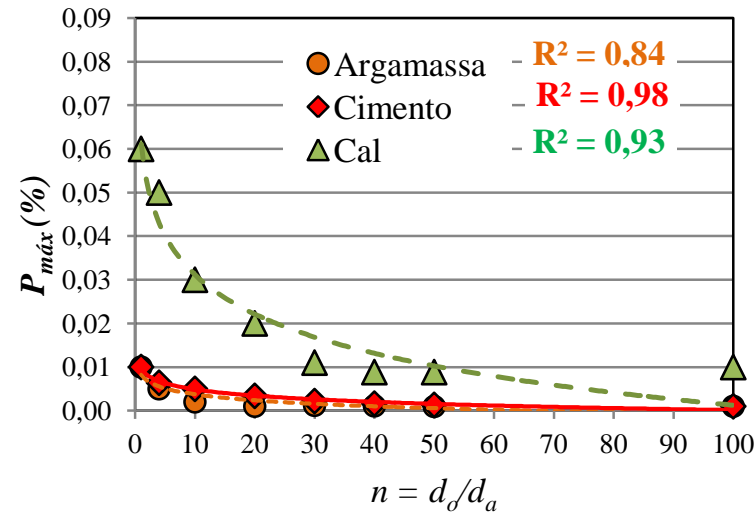

(a) Argamassa, cimento e cal

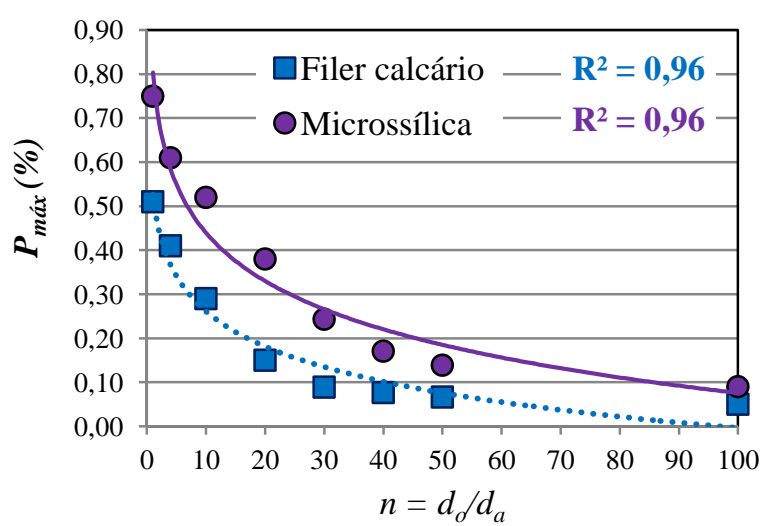

(b) Fíler calcário e microssílica

Figura 4 - Potencial de penetração de partículas em função de $n=d_{o} / d_{a}$

Nota: $d_{o}$ é o diâmetro dos poros do substrato cimentício, e $d_{a}$ o diâmetro das partículas presentes nas matrizes estudadas.

Para $n=1$, a matriz de microssílica preenche $62,44 \%$ (SCIM-A), 23,23\% (SCER-B) e 24,37\% (SCER-C); e para $n=10$, a porcentagem diminui para $43,12 \%, 3,02 \%$ e $9,54 \%$ respectivamente. Nos casos analisados, o volume de partículas que penetra nos poros é limitado pelo volume de poros disponíveis. A penetração ocorre nos poros com diâmetro superior a $0,1 \mu \mathrm{m}$; e não há penetração em poros com diâmetro inferior a $0,1 \mu \mathrm{m}$, visto que não há partículas com diâmetro inferior a $0,1 \mu \mathrm{m}$, tal como mostra a Figura 5 .
Para as condições do modelo adotado e as combinações de matrizes e substratos estudados, verificou-se que a massa de partículas dentro dos poros é desprezível (Tabela 1), o que leva a acreditar que o mecanismo de intertravamento mecânico pela penetração de partículas nos poros não pode ser considerado como o único responsável pela aderência matriz-substrato.

A aderência está relacionada à área de contato matriz-substrato: quanto menor a taxa de defeitos, maior a aderência. Todo defeito reduz a aderência. 

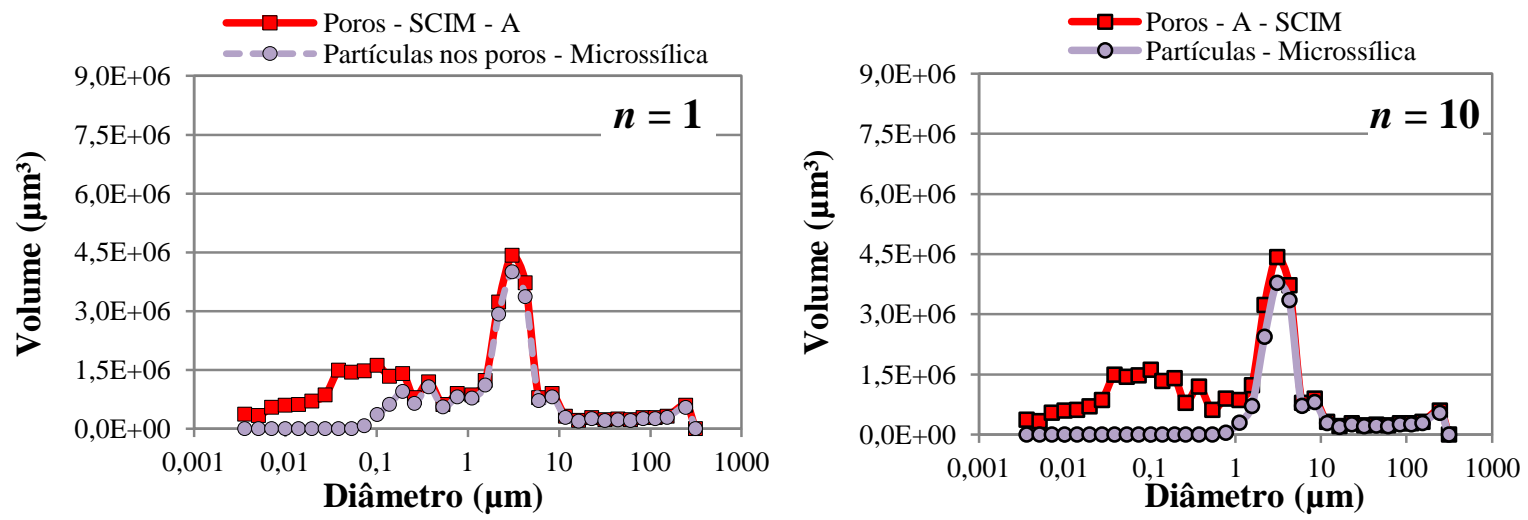

(a) Substrato cimentício - SCIM-A
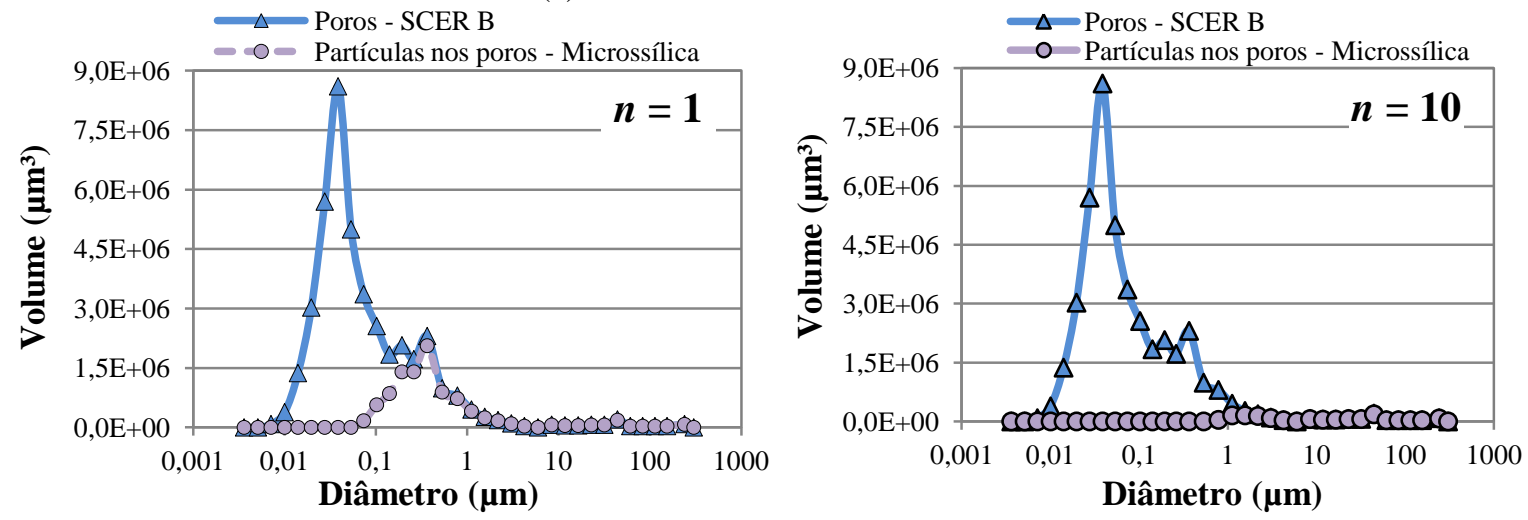

(b) Substrato cerâmico - SCER-B
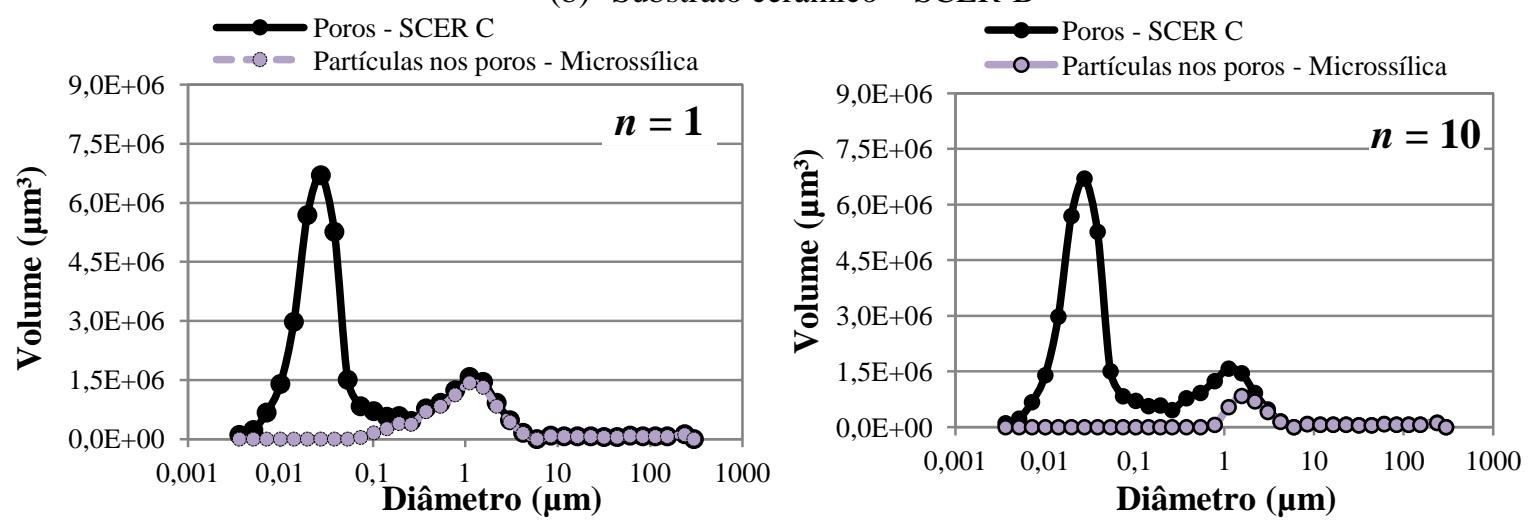

(c) Substrato cerâmico - SCER-C

Figura 5 - Volume de poros do substrato e volume partículas de microssílica nos poros do substrato

Segundo Kendall (2001), a aderência é elevada quando duas superfícies estão em contato molecular, mas a separação de $1 \mathrm{~nm}$ reduz a aderência significativamente. Os poros do substrato são descontinuidades da superfície e, quando eles são preenchidos, há um ganho potencial de aderência (eliminação dos defeitos). $\mathrm{O}$ acréscimo de área de contato pelo preenchimento dos poros dos sistemas analisados é apresentado na Tabela 1.

Nas combinações substrato-argamassa, o acréscimo da área de contato não ultrapassa $0,2 \%$, enquanto a matriz constituída por microssílica proporciona um aumento de 21,48\%, 9,66\% e $8,80 \%$ para os respectivos substratos: SCIM-A, SCER-B e SCER-C. Com base nesses resultados, pode-se concluir que a incorporação de partículas finas em matrizes cimentícias, desde que estas estejam dispersas, pode aumentar o potencial de recobrimento da superfície do substrato, diminuindo os defeitos e a porosidade do sistema.

\section{Considerações finais}

A capacidade de penetração de partículas presentes em matrizes constituídas por cimento, cal, finos e argamassa nos poros de substratos com 
distribuição de poros similares aos encontrados no mercado nacional foi calculada considerando-se a relação entre a área do poro e a área das partículas, admitindo-se que somente partículas com área de projeção inferior à área do poro irão penetrá-lo.

$\mathrm{O}$ potencial de penetração foi pequeno, não atingindo $1 \%$ do volume de partículas totais presentes na matriz, mesmo para a matriz constituída de partículas mais finas (microssílica) em substrato com maior dimensão (cimentício). $\mathrm{O}$ volume de partículas que pode penetrar nos poros é limitado pelo volume de poros do substrato.

Os resultados obtidos indicam que a quantidade de material nos poros é pequena, mesmo se todos os poros forem preenchidos por partículas. Assim, não se pode atribuir unicamente à ancoragem mecânica pela penetração de partículas nos poros a responsabilidade pela aderência substrato-matriz. Vale ressaltar que o modelo proposto considerou a melhor das hipóteses de penetração: desprezando os efeitos de aglomeração e colisão das partículas e admitindo a alocação ótima das partículas nos poros, o que não ocorre nos sistemas reais.

O modelo mostra que matrizes com partículas finas apresentam maior potencial de penetração. Supõe-se que a utilização destas pode diminuir a porosidade e os defeitos na interface e na matriz, pelo efeito de empacotamento, consequentemente ampliando a aderência.

\section{Referências}

BAGLIONI, P.; GIORGI, R. Soft and Hard Nanomaterials For Restoration and Conservation of Cultural Heritage. Soft Matter, v. 2, n. 4, p. 293, 2006.

BORISOVA, E.; ADLER, P. Deposition in Porous Media and Clogging on the Field Scale. Physical Review E, v. 71, n. 1, 2005.

CARASEK, H. Aderência de Argamassas à Base de Cimento Portland a Substrato Porosos: avaliação dos fatores intervenientes e contribuição ao estudo do mecanismo da ligação. 1996. 285 f. Tese (Doutorado em Engenharia Civil) - Escola Politécnica, Universidade de São Paulo, São Paulo, 1996.

CARDOSO, F. A. Método de Formulação de Argamassas de Revestimento Baseado em Distribuição Granulométrica e Comportamento Reológico. 138 f. São Paulo, 2009. Tese (Doutorado em Engenharia Civil) - Escola Politécnica, Universidade de São Paulo, São Paulo, 2009.
CARDOSO, F. A. Distribuição Granulométrica de Partículas de Cimento ao Longo do Tempo. 2010. Relatório interno Laboratório de Microestrutura e ecoeficiência.

CARVALHO JÚNIOR, A. N. Avaliação da Aderência dos Revestimentos Argamassados: uma contribuição à identificação do sistema de aderência mecânico. 331 f. Belo Horizonte, 2005. Tese (Doutorado em Engenharia Civil) Universidade Federal de Minas Gerais, Belo Horizonte, 2005

CHEVOIR, F.; GAULARD, F.; ROUSSEL, N. Flow and Jamming of Granular Mixtures Through Obstacles. Europhysics Letters (EPL), v. 79, n. 1, p. 14001, 2007.

COSTA, E. B. C.; JOHN, V. M. Aderência Substrato-Matriz Cimentícia: estado da arte. In: SIMPÓSIO BRASILEIRO DE TECNOLOGIA DE ARGAMASSAS, Belo Horizonte, 2011. Anais... Belo Horizonte: ANTAC, 2011.

DANIELE, V.; TAGLIERI, G.; QUARESIMA, R. The Nanolimes in Cultural Heritage Conservation: characterisation and analysis of the carbonatation process. Journal of Cultural Heritage, v. 9, n. 3, p. 294-301, 2008.

DÉTRICHÉ, C. H. et al. Mouvements d'Eau, Hydratation et Comportement Mécanique des Mortiers d'Enduit. Matériaux et Constructions, v. 17 , n. 100 , p. 297-302, 1984.

DUPIN, I.; DETRICHE, C. H.; MASO, J. C. Accrochage Direct d'un Enduit Sur un Isolant Par Une Liaison de Type Mécanique Dans le Cadre d'un Procédé d'Isolation Par l'Extérieur.

Materials and Structures, v. 21, n. 5, p. 370-378, 1988.

IMDAKM, A.; SAHIMI, M. Transport of Large Particles in Flow Through Porous Media. Physical Review A, v. 36, n. 11, p. 5304-5309, 1987.

JULLIAND, P. et al. Dissolution Theory Applied to the Induction Period in Alite Hydration.

Cement and Concrete Research, v. 40, n. 6, p. 831-844, jun. 2010.

KAISER, C. A Directed Percolation Model For Clogging in a Porous Medium With Small Inhomogeneities. Transport in Porous Media, v. 26, n. 2, p. 133-146, 1997.

KAMPEL, G. Mathematical Modeling of Fines Migration and Clogging in Porous Media. Tesis - Georgia Institute of Technology, 2007.

KENDALL, K. Molecular Adhesion and Its Applications: the sticky universe. $2^{\text {nd }}$ ed. New York: Kluwer Academic; Plenum Publishers, 2001. 
MAYS, D. C. Contrasting Clogging in Granular Media Filters, Soils, and Dead-End Membranes. Journal of Environmental Engineering, v. 136, n. 5, p. 475, 2010. A

MCDOWELL-BOYER, L. M.; HUNT, J. R.; SITAR, N. Particle Transport Through Porous Media. Water Resources Research, v. 22, n. 13, p. 1901-1921, 1986.

REGE, S. D.; FOGLER, H. S. Network Model For Straining Dominated Particle Entrapment in Porous Media. Chemical Engineering Science, v. 42, n. 7, p. 1553-1564, 1987.

RIPPERGER, S. Crossflow Microfiltration: state of the art. Separation and Purification Technology, v. 26, n. 1, p. 19-31, 2002.

ROUSSEL, N.; NGUYEN, T.; COUSSOT, P. General Probabilistic Approach to the Filtration Process. Physical Review Letters, v. 98, n. 11, 2007.

SCRIVENER, K. L.; CRUMBIE, A. K.; LAUGESEN, P. The Interfacial Transition Zone (ITZ) Between Cement Paste and Aggregate in Concrete. Interface Science, v. 12, n. 4, p. 411 421, 2004.
SHARMA, M. M.; YORTSOS, Y. C. Fines Migration in Porous Media. AIChE Journal, v. 33, n. 10, p. 1654-1662, 1987.

SILVA, D.; MONTEIRO, P. Analysis of $\mathrm{C}_{3} \mathrm{~A}$ Hydration Using soft X-Rays Transmission Microscopy: effect of EVA copolymer. Cement and Concrete Research, v. 35, n. 10, p. 20262032, 2005.

STROEVEN, P.; HU, J. ITZ's Structural Evolution During Hydration in Model Concrete. Magazine of Concrete Research, v. 61, n. 5, p. 371-377, 2009.

TAVARES, M. L.; VEIGA, M. do R. A Conservação de Rebocos Antigos: restituir a coesão perdida através da consolidação com materiais tradicionais e sustentáveis. In: SIMPÓSIO BRASILEIRO DE TECNOLOGIA DE ARGAMASSAS, Belo Horizonte, 2011. Anais... Belo Horizonte: ANTAC, 2011.

\section{Agradecimentos}

Ao Prof. Rafael G. Pileggi (Poli-USP), pelas considerações, e à FAPESP (processo FAPESP $n^{\circ}$ 2010/10843-7) e à CAPES, pelo apoio financeiro.

Revista Ambiente Construído

Associação Nacional de Tecnologia do Ambiente Construído

Av. Osvaldo Aranha, $99-3^{\circ}$ andar, Centro

Porto Alegre - RS - Brasil

CEP 90035-190

Telefone: +55 (51) 3308-4084

Fax: +55 (51) 3308-4054

www.seer.ufrgs.br/ambienteconstruido

E-mail: ambienteconstruido@ufrgs.br

34 Costa, E. B. C.; Kurokawa, F. A.; John, V. M. 